\title{
¿Cómo aprender a leer en una sociedad inclusiva? Contextos internacional y nacional
}

How to learn to read in an inclusive society? International and national contexts

\author{
Carlos Rosales López (iD \\ e-mail: carlos.rosales@usc.es \\ Universidade de Santiago de Compostela. España
}

\section{Resumen}

En estos momentos y a nivel universal, son muchas las personas que no pueden acceder al aprendizaje de la lectura o que si lo hacen deben aprender a leer en una lengua diferente a la propia. Este trabajo tiene por objeto analizar las características que deberían presentar la enseñanza y el aprendizaje de la lectura en una sociedad inclusiva. Se plantean seis interrogantes al respecto y se perfilan posibles respuestas. Para responder a la primera pregunta se utilizan referencias internacionales. En las siguientes se hace referencia a investigaciones y datos centrados de forma predominante en nuestro país. Para su realización se ha hecho uso de importantes documentos de organizaciones internacionales como la ONU y la UNESCO. También, de documentos legales y administrativos. Desde una dimensión empírica se ha utilizado la observación naturalista y el relato autobiográfico, así como la entrevista semiestructurada e instrumentos para el análisis de libros de lectura. Estas técnicas e instrumentos se han utilizado por maestros en formación del Grado de Educación Primaria durante varios cursos, especialmente el 2017-2018 en periodos de prácticum y clases interactivas. En los resultados se subraya la necesidad de extender el aprendizaje en lengua materna, de fomentar un aprendizaje plural en cuanto a soportes y contextos, de intensificar y diversificar las actividades de promoción y animación a la lectura, de adaptar las características de los libros y fomentar hábitos lectores, de cuidar las actividades de lectura en las aulas.

Palabras clave: sociedad inclusiva; aprendizaje de la lectura; animación lectora; libros de lectura; hábitos lectores; lectura en el aula.

\section{Abstract}

Nowadays and at a universal level, there are many people who cannot access learning to read or who, if they do, must learn to read in a language different from their own. This work aims to analyze the characteristics that should be presented in the teaching and learning of reading in an inclusive society. Six questions are raised in this regard and possible answers are outlined. International references are used to answer the first question. In the following ones, reference is made to research and data predominantly centered in our country. Important documents of international organizations such as the UN and UNESCO have been used to create this paper, as well as legal and administrative documents. From an empirical dimension, naturalistic observation and autobiographical narrative have been used, as well as semi-structured interviews and tools for the analysis of book reading. These techniques and instruments have been used by teachers in training for the Primary Education Degree during several courses, especially 2017-2018 in practicum periods and interactive lessons. The results emphasize the need to extend learning in the mother tongue, to encourage a plural learning in terms of supports and contexts, to intensify and diversify the activities of promotion and animation to reading, to adapt the characteristics of books and encourage reading habits, and to take care of reading activities in the classroom.

Keywords: inclusive society; learning to read; reading animation; reading books; reading habits; reading in the classroom.

Recibido / Received: 19-10-2019

Aceptado / Accepted: 01-01-2020

Publicación en linea / Published online: 03-03-2020

Cómo referenciar este artículo / How to reference this article:

Rosales, C. (2020). ¿Cómo aprender a leer en una sociedad inclusiva? Contextos internacional y nacional. Tendencias Pedagógicas, 36, pp. 141-153. doi: 10.15366/tp2020.36.11 


\section{Introducción}

En este trabajo se parte del concepto de sociedad inclusiva como aquella que hace partícipes a todos sus miembros de los mismos derechos y oportunidades con independencia de sus características. La entidad más representativa de la promoción de una sociedad inclusiva es la ONU, que desde la Proclamación Universal de los Derechos Humanos en 1948, estimula de forma constante su extensión a todas las personas en todos los lugares del mundo. Entre las instituciones creadas por la ONU que se destacan en este esfuerzo, se encuentra la UNESCO, que proyecta su intervención en el ámbito educativo. Una sociedad inclusiva supera las barreras que separan unos grupos de otros en función de características como cultura, raza o sexo. Procura el desarrollo de todos ellos no en compartimentos separados, sino a través de la comunicación, la interacción y la colaboración. Trata de desarrollar vínculos de solidaridad. (Kymlicka, 1995; Zeichner, 2010; Banks, 2015)

Una sociedad inclusiva promueve una educación inclusiva, en la que niños y niñas con distintas culturas, lenguas, capacidades o estatus socioeconómicos puedan progresar juntos en el ámbito del aprendizaje y la maduración. Una sociedad no es inclusiva cuando permite que muchos niños y niñas no estén escolarizados o los escolariza de forma precaria discriminando a niñas y discapacitados/as. Tampoco es inclusiva sino asimiladora cuando obliga a sus alumnos/as a aprender a comunicarse en una lengua diferente a la que les es propia, dificultando su acceso a la cultura materna, con la que han tomado contacto de forma oral en el hogar desde los primeros momentos de su vida. (Fernández, 2009; Arnaiz y Guirao, 2015: Casanova, 2016)

Un análisis, aunque sea breve, de disposiciones a nivel internacional, europeo y nacional, pone de relieve la existencia de un constante paralelismo entre disposiciones sociales y educativas de carácter inclusivo. En ellas se registra un cambio cualitativo muy significativo desde el concepto de integración al de inclusión. Es evidente que en sociedades democráticas, como explica Banks (2015, 17 18), se debe superponer la convivencia en igualdad a la simple asimilación como imposición de la cultura mayoritaria a las minorías. Dentro de la amplia serie de normas que a nivel internacional apoyan esta línea argumentativa, A. Casanova (2016, 30-45) cita las siguientes: la Declaración de los Derechos del Niño (1959); la Declaración de las Naciones Unidas sobre la eliminación de todas las formas de discriminación racial (1963); la Declaración sobre los Derechos Humanos de los individuos que no son nacionales del país en que viven (1985); la Convención de las Naciones Unidas sobre los Derechos del Niño (1990). En el ámbito más próximo de Europa existen disposiciones propias, en el sentido de extender el derecho a una educación de calidad a todos los ciudadanos. De manera especial se puede hacer referencia al Libro Blanco sobre la Educación y la Formación (1996) y al Dictamen del Comité Económico y Social sobre «Una educación de alta calidad para todos» (2017). En el contexto español, en el que se proyectan la mayoría de las referencias que en este trabajo se realizan sobre el aprendizaje de la lectura, se podría citar una enorme cantidad de disposiciones a través de las cuales nuestro país muestra su aceptación y cumplimiento de normativas internacionales y europeas. Desde la Constitución de 1978, todas las leyes orgánicas de educación (LODE, 1985; LOGSE, 1990; LOE, 2006; LOMCE, 2013), así como numerosos decretos y órdenes ministeriales y dentro de cada Comunidad Autónoma la normativa propia, especialmente en aquellas Comunidades en las que existe una lengua cooficial.

Así pues, existe una diversidad de contextos en el terreno de la inclusión social y educativa. En este trabajo se utilizarán de forma combinada las referencias a un contexto internacional y al ámbito más próximo de nuestro país. Las diferencias disminuyen y se confunden en la actualidad cuando observamos la presencia en Europa, y de manera específica en España, de minorías autóctonas e inmigrantes con culturas y lenguas propias.

En este trabajo se parte del supuesto de que el aprendizaje de la lectura comprensiva es fundamental para el desarrollo de una ciudadanía inclusiva porque facilita el acceso a la información, haciendo posible una mejor comprensión crítica de la realidad y un ejercicio más responsable de los propios derechos y obligaciones. Probablemente ha sido Paulo Freire uno de los pedagogos que más intensamente han vivido y desarrollado este principio ya que defiende en su método de enseñanza de la lectura a personas adultas la utilización desde el comienzo de palabras que tienen gran significado para ellos, que pertenecen a su entorno cultural. Posiblemente se inspiró en la forma en que su padre le enseñó a leer, escribiendo en la arena con un trozo de madera palabras de su universo familiar. (Gerbardt, 1994)

Se considera en este sentido como hipótesis de investigación que el aprendizaje eficaz de la lectu- 
ra debe realizarse en la lengua materna, ya que esta constituye el vínculo necesario con la realidad conocida por el niño/a desde los primeros momentos de su vida y puede hacer más posible la comprensión. Complementarias con esta hipótesis se consideran las siguientes: a) desde un primer momento se debe estimular un aprendizaje rico y ampliado de lectura que comprenda la actividad oral, la silenciosa y la lectura en diversos soportes, no solo impreso, sino también digitales, b) la promoción de la lectura constituye una tarea de la comunidad globalmente considerada, lo que implica la coordinación de instituciones, personas y programas, así como la dotación de medios adecuados, c) se propone finalmente como hipótesis también, la posibilidad de estimular hábitos de lectura comprensiva en los diferentes contextos de vida del niño/a, así como de perfeccionar las prácticas de lectura en las aulas. En el análisis que se realiza del aprendizaje de la lectura, se toma en consideración el carácter multicultural de nuestro país, en el que además de lenguas mayoritarias, existen al tiempo lenguas minoritarias.

\section{Interrogantes sobre la lectura}

En relación con las hipótesis formuladas, para abordar el tema de la lectura infantil en una sociedad diversificada e inclusiva, me he planteado seis preguntas, a las que trato de responder de manera no exhaustiva, sino mediante la realización de propuestas que considero que pueden ser constructivas.

\section{1. ¿En qué lengua aprender a leer y escribir?}

En el Año Internacional de las Lenguas Indígenas, 2019, resulta fácil responder a esta pregunta. Organizaciones internacionales como la ONU y la UNESCO apoyan fuertemente el aprendizaje de la lectura y la escritura en la lengua materna, aquella en la que las personas adquieren sus primeros conocimientos de forma oral en el hogar y la comunidad. Sin embargo, los aprendizajes de la lectura y la escritura no están universalmente extendidos. Ya en el Informe de la UNESCO coordinado por J. Delors (1996,) se consideraba que, en torno a 128 millones de niños y niñas en edad de asistir a la escuela, no estaban escolarizados. Además, muchos otros millones de niños y niñas que sí asisten a la escuela lo hacen durante escasos periodos de tiempo, insuficientes para lograr un aprendizaje eficaz de la destreza lectora. A estos datos correspondientes a la infancia, hay que agregar la existencia aun en nuestros días de muchos millones de personas adultas que no saben leer ni escribir. No se han cumplido, por lo tanto, los objetivos que la ONU se fijó en la Conferencia de Dakar del año 2000 para el 2015. Entre ellos estaba la extensión de la educación básica a toda la población infantil y la reducción en un $50 \%$ de la tasa de analfabetismo adulto. En la actualidad la UNESCO se propone, de cara al año 2030, una extensión de la educación obligatoria de al menos diez años a toda la población del mundo, así como la erradicación del analfabetismo adulto.

Durante mucho tiempo, las poblaciones indígenas han estado marginadas. A ellas se asocian los índices más graves de depresión socioeconómica. Sus culturas han carecido del necesario reconocimiento y los servicios educativos han sido inexistentes o han constituido un instrumento para la imposición de la cultura mayoritaria. Desde una perspectiva sociocultural se produce un cambio importante con origen en la segunda mitad del siglo XX, a partir del reconocimiento internacional de sus derechos fundamentales. En el ámbito nacional se reconocen también de manera progresiva sus derechos sociales, políticos, culturales y educativos y se manifiesta una importante evolución desde la antigua homogeneidad sociocultural a un progresivo reconocimiento de la diversidad y de las relaciones de interculturalidad. La Asamblea General de las Naciones Unidas proclamó 2019 como Año Internacional de las Lenguas Indígenas, siendo la UNESCO coordinadora del mismo. Con ello pretende crear sensibilidad en torno a la situación de miles de lenguas en riesgo de extinción y fomentar medidas de apoyo para su conservación. Al hacerlo manifiesta que:

«La mayoría de las siete mil lenguas que se hablan en el mundo se consideran indígenas. Todas ellas son depositarias y portadoras de cultura, conocimiento, valores e identidad. Su pérdida representa un empobrecimiento para la humanidad en su conjunto y para las comunidades a las que se impide transmitir su lengua a sus hijos.» (UNESCO, 2019, 1)

La Unión Europea constituye un área eminentemente compleja desde una perspectiva cultural y lingüística. La conformación de la ciudadanía europea, que hunde sus raíces en los principios demo- 
cráticos de los tratados de Roma, Ámsterdam y Maastricht, se ha hecho operativa a través de convenios como los de libre circulación y el derecho al voto en el propio municipio. El incremento de la movilidad de las personas dentro de los países de la Unión, el intercambio de productos, la realización de proyectos en común, han incrementado los contextos de interacción entre culturas y lenguas. Desde una perspectiva lingüística, la complejidad es enorme. En Europa se hablan las lenguas oficialmente reconocidas de los países que la constituyen. Se hablan asimismo lenguas minoritarias autóctonas dentro de dichos países. Unas tienen reconocimiento oficial como en España el vasco, el catalán y el gallego y otras no están reconocidas oficialmente por sus propios estados, como ocurre en Francia y Grecia. Existen también comunidades minoritarias que hablan lenguas oficiales de algún país de la Unión pero están ubicadas en otro. Ocurre así, por ejemplo, con comunidades de gente que habla alemán y vive en Italia o Francia. Hay que subrayar, por otra parte, la existencia de minorías inmigrantes que se han introducido en la Unión Europea procedentes de otras regiones o continentes, como las comunidades turca y subsahariana. Existen asimismo minorías con características peculiares, como la de los gitanos, presentes durante mucho tiempo en la Unión Europea. Ante esta compleja realidad cultural y lingüística, la Unión ha reaccionado reconociendo la necesidad de formación de las personas en el plurilingüismo, pero poniendo el acento en la conveniencia/necesidad de dominio por cada ciudadano de más de una lengua de las mayoritarias y oficiales en la Unión. Así, en el Libro Blanco sobre la Educación y la Formación (1996, pp. $10-$ 11), se proponen cinco orientaciones y concretamente, en la cuarta se dice:

\begin{abstract}
«Dominar tres lenguas comunitarias: un sello de calidad. El dominio de varias lenguas se ha convertido hoy en día en una condición indispensable para la obtención de un empleo. Esto es tanto más verdadero en un mercado sin fronteras, pero es también un triunfo que permite dirigirse a los otros más fácilmente, descubrir culturas y mentalidades diferentes, estimular la propia agilidad intelectual.»
\end{abstract}

Veinte años después, en el Dictamen del Comité Económico y Social Europeo sobre «Una educación de alta calidad para todos» (2017) se intensifica el compromiso con la extensión universal del derecho a la educación y con el cumplimiento de los objetivos de la Unión para 2020 y de las Naciones Unidas para 2030 (art. 1.1), instándose a los «Estados miembros a procurar mayores sinergias y estrategias concertadas a escala de la Unión Europea en el ámbito de la educación para todos» (art. 1.6).

\title{
2.2. ¿En qué consiste la lectura?
}

Se considera que la lectura es una actividad mental muy compleja, en la que a la asociación de signos con significados se sobrepone una importante actividad de análisis, reflexión e imaginación. La persona que lee reconstruye de forma constante la comprensión del texto a medida que avanza en sus contenidos, los asocia con conocimientos previos y los proyecta imaginativamente. De aquí la importancia de que el aprendizaje de la lectura se realice en la lengua materna, aquella que el niño/a ha aprendido ya oralmente en su hogar y que ha sido vehículo de la cultura de su comunidad. Podríamos hacer referencia a la alegoría de Rodari (2002, p. 13), cuando compara los efectos de una palabra en nuestro pensamiento con las ondas que una piedra origina en un estanque:

«Una china tirada a un estanque que suscita ondas concéntricas que se extienden sobre su superficie, involucrando en su movimiento, a distancias distintas, con distintos efectos, al nenúfar y al junco, al barquito de papel y a la balsa del pescador. Objetos que se mantenían a su aire, en su paz o en su sueño, son como reclamados a la vida, obligados a reaccionar, a entrar en relación entre sí.»

Existen básicamente dos importantes modalidades de lectura: oral y silenciosa. La primera tiene vocación grupal y la segunda, autónoma e individual. Ambas modalidades deberían fomentarse desde un principio, pero tanto desde una perspectiva social, como desde una perspectiva pedagógica, la oral antecede a la práctica de la lectura silenciosa. El proceso lector es cualitativamente diferente en ambas. En la oral, el lector asocia los signos gráficos a los sonidos y estos a los significados. En la silenciosa se potencia una asociación directa de signos gráficos con los significados correspondientes. Por ello se considera que en la lectura silenciosa se gana en rapidez y comprensión 
lectora.

En el ámbito escolar, actualmente, la práctica de la lectura oral ocupa un lugar preeminente al comienzo del aprendizaje lector y como eje central de numerosas actividades de aprendizaje. De hecho, el primer contacto del niño con textos escritos y su lectura suele tener lugar en la etapa de escolaridad infantil, a través de actividades como «la hora del cuento». La maestra/o elige libros infantiles que conectan con importantes centros de interés de los niños/as y procede a su lectura oral, en lo que en realidad se convierte en una representación grupal participativa. La hora del cuento constituye, en realidad, una actividad muy compleja de enseñanza y aprendizaje, en la que intervienen no solo el lenguaje oral, sino también el uso de lenguajes paraverbales (ritmo, tono, volumen...) y no verbales (gesto, mirada, movimiento). Toda esta gama de actividades y lenguajes se proyecta finalmente en el reconocimiento por el niño del valor de los textos escritos y su lectura e incrementa notablemente su motivación hacia el aprendizaje lector.

En la escuela se fomenta también de manera progresiva la práctica de la lectura individual y silenciosa, con incremento de la reflexión, acomodada a estrategias personales del alumno/a y al servicio de la realización de muy diversos aprendizajes, así como del enriquecimiento cultural personal y el entretenimiento. De manera específica, se puede realizar la distinción entre lectura oral, lectura silenciosa y lectura de seguimiento. Normalmente en situaciones grupales como las que se dan en las aulas, cada alumno/a suele leer una parte de un texto en voz alta, mientras que sus compañeros/as realizan al tiempo una lectura de seguimiento del mismo. Al parecer, según ciertas investigaciones (García-Rodicio, Melero, \& Izquierdo, 2017), la lectura oral presenta unas características semejantes en cuanto a nivel de comprensión a la lectura silenciosa. No así la de seguimiento, en la que los alumnos/as han de esforzarse por prestar atención a su compañero/a que lee en voz alta, y ese esfuerzo da lugar a un menor rendimiento en comprensión lectora.

En el momento actual niños y niñas, jóvenes y adultos practicamos de manera creciente la lectura en pantallas de televisión, ordenador, móviles, e-books, tabletas. La versión digital de una cantidad creciente de obras extiende considerablemente las posibilidades de lectura, que con los textos convencionales se limitan en función de condicionantes como almacenamiento, transporte, acceso a bibliotecas alejadas, etc. La nueva forma de lectura en pantallas presenta características cualitativamente diferentes a la lectura tradicional con libros impresos (Levattro, 2017). El soporte digital hace posible una utilización diversificada de múltiples lenguajes: el de la palabra escrita, pero también el de la imagen y el de la voz y el sonido. La digitalización se presta a un mayor protagonismo de la imagen estática o dinámica y el acompañamiento de la voz o la música convergen con el texto escrito en la lectura. Probablemente, el diferente protagonismo de estos lenguajes repercutirá también en una diferente estimulación de la inteligencia conceptual (más ligada a la palabra) y emocional (más ligada a la imagen). En la actualidad existe en nuestro entorno una amplia y creciente producción editorial de obras digitalizadas, a las que se puede acceder a través de diversas páginas web, como la creada por Lafarga (http://edu.symbaloo.com/mix/cuentosblafarga). Se trata de cuentos interactivos para educación infantil, en los que el niño/a puede seguir o elegir alternativas al desarrollo de la historia manejando determinados iconos (Martin, 2015).

Los contextos en los que tiene lugar la lectura ejercen una gran influencia sobre las características de la misma. En primer lugar, el familiar, en el que se dan los primeros pasos de una lectura en gran parte motivadora y de entretenimiento, fuertemente vinculada con la cultura oral. Padres y familiares en general, pueden inducir una actitud positiva en los niños/as hacia la lectura proporcionando espacios, tiempos y recursos, pero, sobre todo, a través de actividades compartidas. También desde el hogar se pueden entablar relaciones de interés con instituciones como ludotecas y bibliotecas infantiles. La influencia del contexto familiar y social en las comunidades minoritarias/indígenas puede desempeñar un importante papel en el aprendizaje por los niños/as de la propia cultura y en la iniciación a la lectura y escritura en la lengua materna. Son varios los factores que concurren en que la influencia de estos entornos sea más o menos decisiva. Es evidente en muchos casos la carencia de recursos didácticos adecuados: libros escritos en la lengua minoritaria/indígena, libros y documentos digitalizados en la misma, ludotecas o bibliotecas infantiles, instituciones de apoyo y orientación a los padres, etc. Más importante aún es la preparación de los padres y familiares que pueden inducir conocimientos en los niños/as y, sobre todo, la existencia en ellos de actitudes positivas para estimular el aprendizaje en sus hijos/as. Esta última condición está estrechamente vinculada a la consideración social y cultural de la propia lengua. Cuando esta es muy baja, es frecuente que los propios padres animen a sus hijos/as a utilizar la lengua de la mayoría social, en la creencia 
de que ello les proporcionará más oportunidades profesionales y personales. Puede ocurrir que los padres hablen entre sí una lengua y con sus hijos/as otra.

El contexto escolar toma el testigo a la familia para un desarrollo lector, que en principio presenta características de continuidad con las primeras prácticas orales y progresivamente estimulará el desarrollo de una lectura silenciosa, cada vez más necesaria para el progreso en las tareas de aprendizaje, así como para el entretenimiento y enriquecimiento cultural. El progreso lector en el ámbito escolar está fuertemente vinculado a las características de los proyectos y planes de lectura y al funcionamiento de las bibliotecas escolares. Hay que poner de relieve la necesidad de un cambio cualitativo en el funcionamiento de los centros escolares y sus programas educativos. En el contexto de una educación inclusiva que potencie la interacción enriquecedora entre culturas y lenguas, los planes lectores deben presentar oportunidades para una práctica motivada de la lectura en lenguas minoritarias/indígenas. Y ello implica la previsión de tiempos, espacios y actividades, la disposición de recursos y, sobre todo, la preparación del profesorado y su trabajo en equipo. También en este caso la coordinación con familias de alumnos/as y otras instituciones civiles que trabajan en la promoción de culturas minoritarias/indígenas constituye un factor enriquecedor

\section{3. ¿Cómo promover la lectura?}

Se puede considerar esta tarea como una responsabilidad ampliamente social. Dado que la lectura constituye un eficaz instrumento de información y capacita a las personas para un mejor conocimiento y ejercicio de sus derechos y deberes, es evidente la necesidad de que instituciones sociales y administrativas intervengan en el sentido de fomentar en todas las personas el desarrollo de una capacidad de lectura crítica y responsable. Esta aspiración está lejos de convertirse en realidad a nivel mundial. Más de 100 millones de niños están aún sin escolarizar y varios cientos de millones de niños/as escolarizados acuden pocos años a la escuela, con lo que difícilmente llegan a dominar las competencias básicas de lectura y escritura. A ello hay que agregar los aproximadamente 700 millones de personas adultas que aún en la actualidad son analfabetos.

Iniciativas administrativas como el II Plan para el Fomento de la Lectura (2017-2020) en nuestro país pueden contribuir de manera eficaz a la dinamización de la actividad lectora a través de la coordinación de instituciones y personas, públicas y privadas en entornos educativos, sociales y culturales, apoyo al sector del libro y a libreros, difusión en medios de comunicación social, etc. Este II Plan encuentra apoyo legal en la Ley 10/2007 de 22 de junio, de la lectura, del libro y las bibliotecas, en la que se consideran como componentes básicos de un plan de fomento de la lectura los siguientes:

«La atención a la población infantil y juvenil, los sectores más desfavorecidos socialmente y las personas con discapacidad, la vinculación de los hábitos lectores con el aprendizaje continuo de los ciudadanos de cualquier edad, el papel insustituible de las bibliotecas públicas, escolares y universitarias, la búsqueda del compromiso de los medios de comunicación y la colaboración interministerial (singularmente entre las administraciones responsables de cultura y educación) y con las comunidades autónomas y entidades locales, un aspecto que se manifiesta en su propia integración en el Observatorio de la Lectura y el Libro.» (Plan de Fomento de la lectura 2017-2020, p. 16)

En este Plan se contemplan seis líneas de intervención relativas a:

- Promoción de hábitos lectores. Se proyecta en la comunidad y en la familia, tratando de consolidar los hábitos de lectura en las personas que ya los poseen y de atraer a la actividad lectora a aquellas que no la practican.

- Impulso de la lectura en el ámbito educativo. Se trata de estimular la práctica de la lectura en centros docentes potenciando la ampliación del tiempo dedicado a ella, la formación del profesorado y la creación de materiales didácticos.

- Refuerzo del papel de las bibliotecas en el acceso a la lectura. Se considera que las bibliotecas públicas ponen a disposición de todas las gentes una enorme cantidad de información. Y en este sentido, se tratará de potenciar sus fondos y gestión, así como el acceso en ellas a fuentes digitalizadas.

- Fortalecimiento del sector del libro. Se considera al sector del libro como la principal in- 
dustria cultural, con una producción creciente de obras a nivel nacional e internacional. Se ve necesario organizarlo, potenciar su modernización y se considera asimismo interesante apoyar a las librerías en su valioso papel de motivación y orientación en la lectura.

- Fomento del respeto a la propiedad intelectual. Se considera que es preciso sensibilizar a los lectores sobre el necesario respeto a los derechos de los creadores de las obras que leen en formatos impresos o digitales.

- Mejorar las herramientas de análisis. Se trata de ampliar la información sobre la situación actual de la lectura, el libro y las bibliotecas, tanto desde una perspectiva cuantitativa como cualitativa, profundizando en su conocimiento. El Observatorio de la lectura y el libro desempeñará un destacado papel al respecto.

En un contexto de educación inclusiva e intercultural, la intervención de las administraciones a través de planes como el citado puede desempeñar un papel importantísimo en la valoración social de lenguas y culturas y en la facilitación de su aprendizaje y uso a través de los medios de comunicación, de los centros escolares, de las bibliotecas públicas, de la promoción de publicaciones y la elaboración de recursos didácticos, la coordinación de actividades de diversas asociaciones e instituciones públicas y privadas...

\subsubsection{Actividades de animación a la lectura}

Constituyen un importante factor para la motivación y el desarrollo de la habilidad lectora. Surgidas en las secciones infantiles de las bibliotecas públicas, se han extendido ampliamente en los centros escolares, cuyas bibliotecas suelen desarrollarlas de forma regular. Forman también parte de programas de educación de adultos y se practican en contextos tan específicos como las aulas hospitalarias. En estos momentos se asocia la animación lectora a los centros escolares, pero en realidad puede desarrollarse en muy diversos ámbitos. Así, en el hogar cuando los padres leen o cuentan a sus hijos historias. También en las ludotecas, en las que una de las actividades más frecuentes oscila en torno a contar o leer cuentos o representarlos. También en las secciones infantiles de las bibliotecas públicas, donde en realidad nació la actividad grupal de animación a la lectura.

La puesta en práctica de la animación a la lectura requiere que se den ciertas condiciones organizativas, funcionales y materiales en los centros, pero sobre todo necesita de la dedicación de profesionales que, más allá del estricto cumplimiento de sus obligaciones, posean una buena actitud positiva hacia la lectura, conozcan bien a sus alumnos/as (necesidades, motivaciones, capacidades), tengan una amplia cultura literaria y sean capaces de dedicar tiempos, a veces no remunerados, para programar actividades, coordinarse y trabajar con compañeros, especialistas, padres, desarrollar las actividades en un clima de alegría y motivación, y ser capaces también de reflexionar sobre lo realizado. Algunas manifestaciones significativas de la animación a la lectura se vinculan con:

- Cuentos y dramatizaciones. Entre las actividades más practicadas para la animación a la lectura en bibliotecas públicas y escolares se encuentra en la actualidad la lectura y dramatización de cuentos y obras de literatura infantil en general.

- Poesía. La poesía constituye una forma de comunicación en la que se transmiten conocimientos, pero sobre todo sentimientos, aspiraciones, ideales, afectos. Con la poesía ha tomado contacto ya el niño antes de acudir a la escuela, si consideramos dentro de ella multitud de nanas, canciones, villancicos, que de forma oral ha aprendido en su hogar o en el entorno de la comunidad.

- Apoyo audiovisual. El hecho de que los niños/as pasen mucho tiempo ante las pantallas (ordenador, televisión, tableta, móvil) se ha considerado a veces como un obstáculo para la realización de actividades comunicativas, como una limitación de la conversación o de la lectura. Es posible, sin embargo, hacer uso de esta atracción por los medios audiovisuales para vincular el visionado de obras con su versión escrita y estimular en los niños/as y adultos en general el interés por la lectura.

- Lectura con perros. En 1999 la asociación Intermountain Therapy Animals, (ITA), creó el programa Reading Education Dogs Program (READ), que se ha aplicado con éxito en numerosas bibliotecas y escuelas de EE.UU. Desde 2014 este programa se ha introducido 
en las aulas hospitalarias del Hospital San Joan de Déu (2016) de Barcelona, por el Centro de Terapia Asistida con perros (CTAC). En el ámbito hospitalario es especialmente interesante la actuación de los perros como acompañantes de niños o mayores en las salas de espera, durante la realización de intervenciones y en periodos de rehabilitación. Los efectos de relajación, comunicación, seguridad, disminución del dolor, progreso en la realización de actividades, son manifiestos, siempre acompañados de un efecto emocional notablemente positivo.

- Lectura digital. Durante muchos años y aun en la actualidad, se ha asociado la actividad lectora a la disponibilidad de medios impresos, libros. Así, en países europeos, el número de bibliotecas es enormemente superior que en países del África subsahariana. Sin embargo, dentro del modo de lectura digital, se manifiesta en la actualidad una novedosa posibilidad, capaz de reducir desequilibrios tradicionales. Se trata de la lectura en teléfono móvil. En la actualidad, de los 7.000 millones de personas que habitamos el mundo, en torno a 6.000 millones poseemos teléfono móvil.

La UNESCO contempla en estos momentos como una importante estrategia para la alfabetización y el desarrollo de la lectura el uso de móviles. En este sentido, ha realizado una amplia investigación (UNESCO 2015) sobre las características de su utilización en siete países: Etiopía, Ghana, India, Kenia, Nigeria, Pakistán y Zimbabue. Esta investigación abre grandes expectativas de futuro en el uso de la lectura en móviles. El hecho de que la mayor parte de los más de 700 millones de analfabetos adultos sean mujeres y que estas se responsabilicen generalmente de la educación de sus hijos/as, sugiere la necesidad de fomentar un uso más extendido del móvil por las mujeres adultas, pues puede considerarse un valioso instrumento para su formación y la de sus hijos. La actual limitación en contenidos digitales debe dejar paso a un progresivo incremento de los mismos, especialmente adaptados a diversos sectores de población. Estos contenidos, además, deberán presentarse cada vez más en las lenguas propias de los lectores, pues en la actualidad se presentan de forma predominante en inglés.

En síntesis, la promoción de la lectura en lenguas indígenas y minoritarias precisa de una decidida actitud de apoyo por parte de las administraciones de los países que quieran convertirse en sociedades diversificadas e inclusivas. Existe una primera intervención de carácter general, que ponga las bases sobre las que desarrollar pluralidad de actividades. La coordinación de instituciones oficiales y civiles debería potenciar la actuación en el contexto social y cultural con el importante apoyo de los medios de comunicación. La lectura digital en móviles está siendo objeto de creciente interés por las posibilidades que ofrece en contextos tradicionalmente carentes de recursos impresos. A las instituciones sociales convencionales como las ludotecas se suman en la actualidad otras, hasta el momento poco mencionadas, como los hospitales. De manera específica, resulta necesario potenciar la actuación en el contexto familiar, en el que la iniciación a la lectura se apoya en el aprendizaje de la cultura materna. Es importante asimismo el desarrollo de la animación a la lectura en el contexto escolar, en el que recursos como los cuentos y dramatizaciones, la poesía y los medios audiovisuales tienen una enorme proyección.

\section{4. ¿Cómo elegir libros y temas de lectura?}

Por ser el recurso didáctico más utilizado en el desarrollo de una educación intercultural e inclusiva, es necesario promover la elaboración de textos adaptados a las características de los alumnos y de su cultura. Desde una perspectiva formal, en los primeros libros que utiliza el niño cuando comienza a leer se da una estrecha combinación de lenguajes. La imagen ocupa un lugar predominante en extensión y significación y a ella se asocian palabras y expresiones de manera progresiva. A veces los libros también incluyen mensajes sonoros. Y en general se hace uso en ellos de un lenguaje complementario de carácter sensomotor: cualidades atractivas del libro al tacto, facilidad de manipulación (Rosales, 2005).

A medida que el niño/a desarrolla su capacidad lectora, el texto escrito adquiere mayor protagonismo y ocupa mayor espacio en la página del libro. De todos modos, la imagen continuará constituyendo un instrumento de comunicación imprescindible, asociada a la palabra. Seguirá desempeñando claras funciones de motivación (captar el interés por un tema), de sustitución de realidades difícilmente descriptibles a través de la palabra y de estimulación de la actividad intelectual e imagi- 
nativa del lector/a. La importancia comunicativa de la imagen en los libros de lectura da lugar a que nos planteemos la necesidad de educar al niño/a en la lectura de la misma. En este sentido, aprender a leer implica no solo ser capaz de interpretar el texto escrito, sino también la imagen que lo acompaña.

Paralelamente al desarrollo del lenguaje icónico, el lenguaje verbal en los libros de lectura experimentará cambios en cuanto al vocabulario, expresiones y discurso. El vocabulario debe enlazar de forma natural con el que el niño ya domina en el ámbito oral. Los términos de su vocabulario común, de uso cotidiano, deberán constituir puntos de anclaje con los que asociar otros nuevos. Y, al mismo tiempo, se potenciará un enriquecimiento de los matices significativos de los conceptos que ya conocía. De manera progresiva, el vocabulario común del niño/a se ampliará con un vocabulario específico (técnico y abstracto). Es muy importante que los nuevos términos se presenten de forma contextualizada, fuertemente relacionados con otros ya conocidos por él/ella, para facilitar así su aprendizaje natural. Las expresiones verbales, en principio muy sencillas, crecerán paulatinamente en extensión y complejidad y el discurso, que en los primeros momentos es predominantemente coloquial, inductivo y dialogado, solo con el tiempo se irá convirtiendo en un discurso más técnico, deductivo o expositivo.

En la evolución de los libros infantiles de estudio, pero también de lectura, la segunda mitad del siglo XX ha marcado un importante punto de inflexión. Se produce, por una parte, una renovación de contenidos de carácter científico, pero sobre todo social. Instituciones internacionales como la ONU, la UNESCO y la OMS alientan el desarrollo en los libros infantiles de valores relativos a la convivencia pacífica, la conservación del medio, la igualdad o la salud. Desde 1988 el Ministerio de Educación viene realizando estudios anuales sobre las características de la edición de libros en España. Estos estudios dan a conocer la evolución de las publicaciones en general y por sectores, como el infantil y juvenil. De los datos proporcionados oficialmente se deriva la situación preocupante de un sector de lectura caracterizado más por la producción en cantidad y por los modelos internacionales, con cierto olvido de autores propios y de géneros clásicos como la poesía y el teatro. Se perfila de cara al futuro un creciente uso de la lectura digital, que será necesario analizar en comparación con la lectura en soporte impreso, aun predominante en el momento actual.

En la elaboración de libros para la lectura y el estudio adaptados a una sociedad inclusiva, es preciso cuidar su lenguaje verbal e icónico, así como sus contenidos. A través de todos sus componentes, los libros deben ser portadores de valores de convivencia, respetuosos con las culturas, continuadores de los primeros aprendizajes orales de los niños/as, portadores de modelos de igualdad y respeto hacia el medio ambiente. Es preciso cuidar en este sentido la elaboración de los libros de lectura, pero también su selección y uso adaptado.

\section{5. ¿Se producen cambios con el paso del tiempo en las lecturas infantiles?}

Para responder a esta pregunta he tratado de estudiar las características de las obras de lectura y de los hábitos lectores de niños/as de seis a doce años de hace cincuenta años y en el momento actual. En la recogida de datos he utilizado la entrevista semiestructurada, aplicada a personas mayores de sesenta años y a niños/as entre seis y doce años, pertenecientes a un estatus sociocultural de tipo medio, durante el curso 2017-2018. A través de la información proporcionada he intentado conocer cuál es la opinión de los lectores/as sobre sus obras preferidas y cómo las leyeron, desde su acceso a las mismas a los contextos y tiempos de lectura, apoyos y dificultades, así como si se las recomendaban a otras personas y si consideran que han influido de algún modo en sus vidas. A las personas mayores se les ha solicitado, además, información sobre diferencias entre las formas de leer en su infancia y en la actualidad. Entre las obras leídas debían elegir una como más apreciada y explicar características de su desarrollo, del contexto en que tiene lugar la acción y de los protagonistas. También se les ha preguntado sobre valores implícitos. Si queremos destacar aspectos específicos, podemos hacer referencia a los siguientes:

Las obras de lectura de niños/as hace cincuenta años pertenecían de manera predominante a la literatura clásica, con temas de interés infantil, pero también universal. Las obras que leen los niños/as en la actualidad se vinculan a los intereses de la edad combinando componentes imaginativos con características de la vida cotidiana. Las formas de conocimiento y acceso a las obras de lectura siguen siendo prácticamente las mismas: la propia familia y la escuela. Los niños/as antes leían de forma más esporádica y puntual, en la medida en que se veían libres de otro tipo de tareas. 
Los niños/as en la actualidad parecen disponer de más tiempo y su lectura puede ser más continua, si bien compaginan la lectura con otras formas de entretenimiento.

Tanto antes como ahora los niños/as entienden el lenguaje de los libros y utilizan estrategias semejantes en el caso de tener dudas. Sin embargo, la letra en los libros de antes era más pequeña y las imágenes, más escasas y generalmente en blanco y negro. Tanto antes como ahora solían comentar las obras con familiares, amigos/as y compañeros/as. Y en los dos casos piensan que han influido de algún modo en sus vidas. Las obras leídas antes presentan una combinación de historias de niños/as y adultos, mientras que las actuales tratan en su mayoría de historias de niños/as. Los contextos de estas historias son muy diversos tanto en uno como otro caso, combinando características de realidad e imaginación.

Los valores que las personas mayores detectan en sus obras se pueden organizar en varios núcleos. El primero, más numeroso, se compone de valores de amistad, convivencia, solidaridad, lealtad, compañerismo, fidelidad al grupo, agradecimiento, comprensión y empatía. Otro importante grupo de valores gira en torno al amor a la vida, a los animales y al medio ambiente. Finalmente, se identifican dos pequeños grupos de valores: uno en torno a la imaginación y fantasía y otro en torno a la valentía, el esfuerzo y las ansias de vivir. En sentido negativo se han identificado situaciones de machismo y discriminación por género, así como de maltrato a niños/as y animales. Los valores detectados por los niños/as en sus lecturas se organizan también en varios núcleos. El primero, más numeroso, es semejante al primer núcleo de las personas mayores, pues se proyecta en el compañerismo, la amistad, la convivencia, la solidaridad, el juego limpio. Otro núcleo importante de valores se refiere al respeto a las personas: a uno mismo y a los demás, no subestimar a otros, practicar la igualdad. A continuación, se señalan, con menos frecuencia, otros tipos de valores como el cuidado del medio ambiente, la valentía y la fantasía e imaginación. Destaca de manera especial en los niños/as la frecuencia con que citan los valores del compañerismo y la amistad, así como la menor frecuencia respecto a los mayores de valores como el amor a la vida y a los animales. En sentido negativo, hacen referencia a situaciones de crueldad y violencia.

Hay que advertir que las entrevistas están realizadas en un contexto social muy homogéneo. No obstante, parece necesario que en los libros de lectura se refleje el carácter progresivamente pluricultural e inclusivo de la sociedad, de modo que todos los lectores tengan acceso a valores de inclusión e interacción cultural, y de manera específica, que quienes pertenecen a un grupo minoritario o indígena puedan ver reflejados componentes de sus propias culturas en las obras que leen.

\section{6. ¿Qué incidentes se producen en torno a la lectura en las aulas?}

No siempre se produce un uso del lenguaje en las mejores condiciones para el aprendizaje. El análisis de incidentes escolares (Rosales, 2015) pone de relieve cómo en ocasiones el lenguaje, y específicamente la lectura en el aula, constituye un vehículo de tensiones y enfrentamientos, un factor que incrementa el estrés y deteriora la imagen social de los alumnos e incluso la del propio profesor/a. Los incidentes son sucesos puntuales que perturban el desarrollo «normal» de las actividades en el aula. Aun cuando las más de las veces la comunicación entre profesores/as y alumnos/as se desarrolla de manera previsible y satisfactoria, el estudio de incidentes pone de relieve la necesidad de prever manifestaciones perjudiciales en la comunicación, así como la necesidad de adoptar medidas adecuadas para la superación de estas cuando se producen. La recogida de incidentes es posible a través de la utilización de la observación naturalista. Los profesores/as que interactúan durante largos periodos de tiempo con sus alumnos/as, generalmente los tutores/as, están en condiciones de observarlos en múltiples situaciones y pueden recoger datos sobre su actividad más normal o frecuente, pero también sobre sus actuaciones más difíciles o inesperadas.

Los profesores/as pueden realizar una interpretación contextualizada de los sucesos observados, reflexionar sobre su origen, sobre las consecuencias que han tenido en el aprendizaje y motivación de sus alumnos, sobre la actuación de los protagonistas y, en concreto, sobre su propia intervención y las posibles alternativas a la misma. Y a la reflexión individual puede seguir la realizada en grupo. En este caso, la primera comprensión e interpretación se enriquece con las perspectivas aportadas por los compañeros/as y el profesor/a en clases interactivas.

Durante el curso 2017-2018, maestros/as en formación de la USC han recogido a través de relatos autobiográficos y han analizado en clases interactivas incidentes escolares, algunos de los cuales tienen relación estrecha con el uso del lenguaje verbal y, en concreto, con la lectura. La técnica de 
recogida y análisis de incidentes constituye el resultado de años de trabajo del autor (Rosales, 2015, 2017) en el contexto de clases interactivas con alumnos/as del grado de maestro/a de la USC. Por ejemplo:

Lectura e inclusión

a) El incidente tiene lugar en un colegio público integrado situado en una población inferior a 10.000 habitantes del entorno rural gallego. Se imparten las etapas de educación infantil, primaria y secundaria obligatoria. De manera específica, nos encontramos en un aula de quinto curso de educación primaria, entre cuyos alumnos, uno es de origen marroquí (Omar) y se encuentra en proceso de integración. De nivel socioeconómico bajo, este alumno no es bien aceptado por sus compañeros. En clase de inglés, el profesor pone un ejercicio a la clase y le manda a este niño contestarlo. Omar intenta leerlo pero comete bastantes errores, de modo que el profesor le dice que pare, que todo está mal y que intente leer en inglés y no en un idioma desconocido. Los compañeros se burlaron de él y el profesor no hizo nada por impedirlo. Posteriormente llamó a una niña, que lo leyó correctamente y el profesor volvió a recriminar a Omar comparándolo con la niña y haciendo comentarios sobre lo poco que en su casa se ocupaban de que hiciera las cosas bien. (C. D. F.)

$\mathrm{El}$ incidente produce descenso de autoimagen e imagen social en Omar y una reacción natural de rechazo hacia el centro, en el que además se ha vinculado su rendimiento a la atención de que es objeto por su familia. La integración de niños/as procedentes de otros países y culturas hace necesario un diagnóstico de su nivel de aprendizaje para poder incluirlos en el curso más adecuado. Pero esto no es todo. Probablemente necesitará la ayuda de profesores de apoyo para superar determinadas cuestiones como el dominio del idioma. Los profesores deberían ser sensibles a la situación de estos niños y colaborar con el departamento de orientación en su inclusión. El establecimiento de buenas relaciones de colaboración con sus familias constituye otro importante factor a tener en cuenta. El desarrollo en el grupo de compañeros/as de una sensibilidad de comunicación, respeto y ayuda sería muy beneficioso para Omar y, fundamentalmente, para todo el grupo.

b) El incidente se desarrolla en un colegio público de educación infantil y primaria situado en una población costera de Galicia. En una clase de segundo curso se encuentra recién escolarizada una niña uruguaya cuya madre acaba de encontrar trabajo en la población. En clase de lengua castellana, al tercer día de acudir al colegio, la profesora encargó al grupo de alumnos/as la realización de una composición sobre sus costumbres y gustos. Una vez acabada, tenían que leerla ante sus compañeros/as. La niña uruguaya utilizó en su composición una serie de términos propios de su país, desconocidos aquí. Ello produjo una reacción de burlas y risas en el grupo y en la propia profesora, que se lo tomó a broma y se burló también de la niña diciéndole que tenía que utilizar los términos «correctos». Todo ello produjo una fuerte reacción de rechazo hacia las clases y el colegio en la niña, justo en el momento inicial de escolarización en el mismo. (I. B. V.)

Una clase en la que conviven alumnos/as con distintas culturas constituye un contexto enriquecedor de la formación de todos sus miembros. No ocurre así cuando se considera superior una cultura y se menosprecian otras. La niña en proceso de integración no puede entender por qué motivos su manera de expresarse se considera incorrecta y el desprecio que se manifiesta hacia la misma se transfiere negativamente a la propia persona. Los niños del grupo están perdiendo en esta situación una importante oportunidad para conocer y apreciar otras costumbres, expresiones, formas de vida...

\section{Conclusiones}

En una sociedad que aspire a ser diversa e inclusiva se debe reconocer el derecho de las personas a aprender a leer y escribir en su propia lengua, así como a ampliar su capacidad de comunicación con el aprendizaje de otras lenguas próximas, en un contexto de interacción constructiva. En sociedades pluriculturales y plurilingües como la nuestra, es preciso poner énfasis en el respeto a los derechos de poblaciones minoritarias, en relación a orientaciones y convenios de carácter internacional, europeo y nacional.

En la enseñanza de la lectura es preciso considerar que se trata de una habilidad mental muy compleja en la que se da una estrecha vinculación con la cultura materna, se puede desarrollar de forma oral o silenciosa, utiliza soportes impresos o electrónicos y se ve influenciada por contextos de vida diferentes como el hogar, la escuela y la comunidad... La promoción de la lectura en lengua materna es una responsabilidad, en principio, de las administraciones de cada país. La existencia de 
disposiciones legales y de planes generales para la coordinación de instituciones y personas constituye un punto de partida fundamental. La realización de actividades de animación a la lectura debería entenderse en sentido amplio, extendiéndose a diversos contextos y proyectándose no solo sobre niños/as, sino también en adultos. En el apoyo al aprendizaje de la lectura en una sociedad plural los libros deberían presentar en sus lenguajes y contenidos unas características adaptadas a las culturas de los niños/as que los van a utilizar. Resulta necesario, en este sentido, trabajar en la creación de medios plurales, reflejo de una sociedad también diversa e inclusiva.

Los contextos en los que se realiza la lectura: hogar, escuela, comunidad, deberían estimular el desarrollo en los niños/as de hábitos de lectura crítica y comprensiva, a través de tareas como la sugerencia de obras, la facilitación a su acceso, el comentario de las mismas, la vinculación con importantes valores culturales, el análisis de los contextos, de los protagonistas, del desarrollo de la actividad, etc. En la creación de estos contextos es preciso el fortalecimiento de actitudes positivas en los padres para la práctica de la lectura en el hogar, en los profesores para la estimulación del aprendizaje de lenguas minoritarias juntamente con las de la mayoría, en la sociedad para hacer posible la presencia de estas lenguas en las tareas cotidianas. El análisis de las características de la lectura oral en las aulas pone de relieve la existencia de incidentes o problemas que pueden afectar de forma especial a alumnos pertenecientes a minorías culturales, en función de sus dificultades de adaptación al idioma de la mayoría. Cuando un aula pretende ser auténticamente inclusiva debe procurar un apoyo equitativo a sus alumnos.

\section{Referencias}

Alumnos del grado de Maestro/a en Educación Primaria (curso 2017-2018) que participaron en la recogida y análisis de incidentes: Iván Bello Varela (I. B. V.), Carolina Dobarro Fraga (C. D. F.).

Arnaiz, P. y Guirao, J. M. (2015). La educación intercultural en una escuela inclusiva. Vías para reorientar el camino. En A. Escarbajal (Ed.) Comunidades interculturales y democráticas (pp. 53-62). Madrid: Narcea.

Banks, J. A. (2015). Emigración global, diversidad y educación para la ciudadanía. En A. Escarbajal (Ed.) Comunidades interculturales y democráticas (pp. 15-24). Madrid, Narcea.

Casanova, A. (2016). Educación inclusiva: un modelo de futuro. Madrid; Wolters Kluwer.

Comisión Europea (1996). Libro Blanco sobre la Educación y la Formación. Enseñar y aprender. Hacia la Sociedad del Conocimiento. Luxemburgo: Oficina de Publicaciones Oficiales de las Comunidades Europeas.

Delors, J. (1996). La educación encierra un tesoro. Madrid: Santillana/UNESCO.

Fernández, J. M. (2009). Un currículo para la diversidad. Madrid: Síntesis.

García-Rodicio, H., Melero, M. A., \& Izquierdo, M. B. (2017). Una comparación de lectura en voz alta, lectura silenciosa y lectura de seguimiento, ¿Cuál es mejor para la comprensión? Infancia y Arendizaje, 41(1), 138-164. doi: 10.1080/02103702.2017.1364038

Gerbardt, H. P. (1994) Paulo Freire. Perspectivas UNESCO, XXIII(3-4). 10.1007/BF02195128

Levattro, V. (2017). Encuentros entre lectura en papel y lectura digital: Hacia una gramática de lectura en entornos virtuales. Foro de Educación, 15(2)3, 85-100. doi: 10.14516/fde.555

Ley 10/2007 de 22 de junio de la lectura, del libro y de las bibliotecas. BOE 150 de 23 de junio.

Ministerio de Educación, Cultura y Deporte (MECD) (2010). Panorámica de la Edición Española del Libro. Madrid: Secretaría General Técnica. Centro de Publicaciones. Ministerio de Cultura.

Ministerio de Educación, Cultura y Deporte (MECD) (2017). Plan de Fomento de la Lectura 20172020. Recuperado de https://www.mecd.gob.es/prensa-mecd/dms/mecd/prensamecd/actualidad/2017/05/20170503-planlectura/archivo1.pdf

Martín, R. A. (2015). Recursos didácticos en lengua y literatura. Madrid: Síntesis.

Rodari, G. (2002). Gramática de la fantasía. Barcelona: Planeta.

Rosales C. (2001). Plurilingüismo y educación. Innovación educativa, 11, 11-24.

Rosales, C. (2005). Los textos escolares: actividades, contenidos y lenguajes En Rosales, C. (Ed.) Temas para la reflexión y la investigación didáctica. Santiago: Tórculo.

Rosales, C. (2015). Los temas transversales en el aula. Santiago: Andavira.

Rosales, C. (2017). Alumnos, maestros, colegios e incidentes. Santiago: Andavira.

ONU (2007). Declaración de las Naciones Unidas sobre los Derechos de los Pueblos Indígenas. Aprobada por la Asamblea General el 13 de septiembre de 2007. Recuperado de 
https://www.un.org/esa/socdev/unpfii/documents/DRIPS_es.pdf

ONU (2015). Documento Final de la Conferencia Mundial sobre los Pueblos Indígenas, aprobada por la Asamblea General el 22 de septiembre de 2014. Recuperado de https://www.acnur.org/fileadmin/Documentos/BDL/2015/9817.pdf

UNESCO (2000). Marco de acción de Dakar: Educación para todos. Recuperado de https://unesdoc.unesco.org/ark:/48223/pf0000121147_spa

UNESCO (2015). Liderar la agenda mundial. Educación 2030. Recuperado de https://es.unesco.org/themes/liderar-agenda-mundial- educación-2030

UNESCO (2019a). Presentación del Año Internacional de las Lenguas Indígenas. Recuperado de https://es.unesco.org/news/presentacion-del-ano-internacional-lenguas-indigenas-2019

UNESCO/OREALC (2019). Conocimientos indígenas y políticas educativas en América latina. Hacia un diálogo de saberes. Segundo Informe. Santiago de Chile: UNESCO/OREALC/ME.

Unión Europea (2017). Dictamen del Comité Económico y Social Europeo sobre «Una educación de alta calidad para todos». Diario de la Unión Europea 31-V-2017. Recuperado de https://eu.vlex.com/source/doue-23/issue/2017/

Zeichner, K. M. (2010). La formación del profesorado y la lucha por la justicia social. Madrid: Morata. 